\title{
CONGENITAL UNILATERAL ABSENCE OF THE VAS DEFERENS
}

Dear Editor,

I would like to share my experience of a case of congenital unilateral absence of left vas in a serving soldier who reported for vasectomy. Congenital unilateral absence of the vas deferens is a rare anomaly. Incidence is 0.06 to $1 \%$ in healthy men [1]. By itself it has little clinical significance other than an associated $79 \%$ incidence of ipsilateral renal agenesis [2]. Hunter in 1775 first described the unilateral absence of the right vas deferens with bilateral absence of the body and tail of the epididymis [3]. It is a rare cause of male infertility when it is associated with contralateral mesonephric duct abnormality [4].

A 30-years-old serving soldier, apparently healthy, reported for vasectomy. He had three children-two males and one female. On examination left side scrotal vas was not palpable while testis was normal. Right vas, testis and epididymis were normal. During operation right vas was explored under local anaesthesia by single incision in the median raphe of the scrotum. One cm segment of the right vas was resected and the two ends occluded with $2 / 0$ silk sutures. Left side vas was not found. Further exploration revealed normal testis and caput epididymis on the left side. Patient had uneventful postoperative recovery. Patient was further evaluated by USS abdomen, both kidneys were normal. Semen analysis after two months reported no dead/alive spermatozoa.

Vas deferens is the least commonly palpated during routine physical examination and is most commonly assaulted in surgery [3]. It may be absent on one or both sides, and in whole or in part, and in the latter case may fail to establish communication with the epididymis [5]. In the population of those who elect to have a vasectomy, $0.06 \%$ to $0.8 \%$ of patients have a single vas deferens [1]. Eighty percent of patients who have cystic fibrosis have bilateral anomalies or absence of the vas deferens [6].

Embyrologically vas deferens is derived from the mesonephric duct. At the $15 \mathrm{~mm}$ stage ( 7 weeks) of gestation, the ureteral bud separates from the remaining mesonephric duct, which will subsequently differentiate into the ejaculatory duct, seminal vesicles and the ampulla of the vas deferens caudally; the vas in the midpor- tion; and distal two thirds of the epididymis cranially. The penetration of the ipsilateral metanephrogenic blastema by ureteral bud induces the development of the ipsilateral kidney. While disturbance in the development of the mesonephric duct, after separation of the ureteral diverticulum may result in the absence of the vas deferens but a normal ureter and kidney as was seen in this patient.

Routine physical examination in all male patients should include bilateral palpation for the vas deferens. The technique is as follows: The testis and the epididymis are placed on gentle traction with one hand and the first and third fingers of the other ungloved hand are placed anteriorly and posteriorly on the median raphe of the scrotum above the testis and epididymis. These fingers are gently moved laterally, allowing the spermatic cord tissue pass between them. A firm, rubbery structure, the vas deferens should be detected towards the lateral aspect of the scrotal sac.

Unilateral absence of the vas deferens is a significant physical finding that may suggest absence of ipsilateral renal unit. Contralateral abnormalities in the solitary renal unit occur in a significant percentage of such patient.

\section{REFERENCES}

1. Klapporth HJ, Young ID. Vasectomy, vas ligation and vas occlusion. Urology 1973;1: 292-300.

2. Schmidt SS. Techniques and complications of elective vasectomy. Fertil Steril 1966;17:467-82.

3. Donohue RE, Fauver HE. Unilateral absence of the vas deferens $A$ useful clinical sign. JAMA 1989;261:180-2.

4. Goldstein M, Schlossberg S. Men with congenital absence of vas deferens often have seminal vesicles. Urology 1988;140:85-6.

5. Kushwaha SS, Pendse AK. Congenital bilateral absence of vas deferens-an uncommon cause of male infertility. Surg $\mathrm{J} N$ India 1996; $12: 144$.

6. Holsclaw DS. Cystic fibrosis and fertility. Br Med J 1969:3:356.

Lt Col S CHAWLA

Classified Specialsit (Surgery), 172 Military Hospital, C/o 56 APO.

\section{POST INJECTION TUBERCULAR INFECTION}

Dear Editor,

$\mathbf{T}$ This is in reference to the article "Post injection tubercular infection" [1]. I would like to mention a few other reports in the literature of tubercular abscesses occurring at the site of subcutaneous or intramuscular injections [2.3]. Most other reports are nearly limited to one or two examples hence giving an impression that the condition is scarce. In fact, there was an epidemic of inoculation in children following vaccination against Pertussis! [3].

Theoretically, the abscess can occur in either of the two ways. Firstly if the organisms are introduced at the injection site by contaminated injection material or instrument. This primary inoculation is unusually rare. Secondly, the more common pathogenesis occurs in those cases who have recently undergone primary infection and during this early stage of the disease (incubation period) a number of bacilli reach the blood stream, either directly from the initial focus or by the way of regional lymph node or thoracic duct [4]. This sporadic dissemination, also termed as Occult Haematogenous Tuberculosis, takes place for a very short time and is unlikely to continue after delayed hypersensitivity develops. Clinical manifestation during this episode is fever which lasts for a few days. The bacilli are seeded at various organs and may heal completely, may progress to active lesion or may remain quiescent containing viable bacilli to become active again during lowered body resistance. The site of infection act as an area of lowered resistance resulting into seedling and progression of the disease if conditions are appropriate.

In the mentioned patient [1], there is a strong possibility that when the patient had fever and received injections, he was in dissemination stage resulting into seedling of bacilli at the site, rather than direct inoculation as mentioned. Secondly the speed of development of abscess depends upon the tuberculin sensitivity stanus of the patient. If he were positive then he would develop a local abscess. This may come on acutely if he is strongly positive. If negarive, then as the local abscess slowly develops, the regional lympnodes also enlarge and generalised tuberculosis may also follow [2].

Thus post injection tubercular abscess should be considered whenever a chronic local abscess forms at the injection site particalarly if there has been an interval of 2-3 weeks between injection and the development of complications. Mantoux test and chest $\mathrm{X}$-rays are again mandatory in such cases.

\section{REFERENCES}

1. Harjai MM, Bajpai RK, Chaturvedi AK. Maudar KK. Deshpande GL. Post injection tubercular infection. MJAFI 1999:55:77-8.

2. Miller FJW. Tuberculosis in children. Ist edition New Delhi. BI 